\title{
The Interplay Between Hardware And Control System Design In The Development Of The Active Rack Isolation System
}

\author{
Dr. Ian J. Fialho' \\ Dynacs Engineering - Boeing ISS \\ Mail Stop HS-44 \\ 2100 Space Park Drive \\ Houston, TX 77058 \\ jan.fialho@sw.bocing.com \\ Dr. Sreekumar Thampi \\ Boeing North American \\ Mail Stop HS-24 \\ 2100 Space Park Drive \\ Houston, TX 77058 \\ .. sreekumar.thampi@sw.boeing.com
}

\section{EXTENDED ABSTRACT}

A primary mission of the International Space Station (ISS) is to provide a premier microgravity laboratory environment for conducting acceleration sensitive scientific research. In order to accomplish this goal, vibroacoustic disturbances caused by station activities that occur during the microgravity mode of operation, must be controlled. In addition to source isolation and other passive isolation methods, the ISS uses active isolation at the receiver, through the use of an Active Rack Isolation System (ARIS), as part of its overall vibration isolation strategy. A schematic diagram of a typical ARIS payload rack is shown in Figure 1. The ARIS isolation control system senses rack acceleration via three triaxial accelerometer heads (labeled 3) and uses eight pushrod actuators (labeled 5) to perform active vibration attenuation. Position sensors housed in the actuator assembly are used to sense the relative position between the rack and the station. Electrical power, data and other essential resources are routed through a set of umbilicals that interface with a passthrough panel at the bottom of the rack. A representative umbilical set is shown in Figure 2.

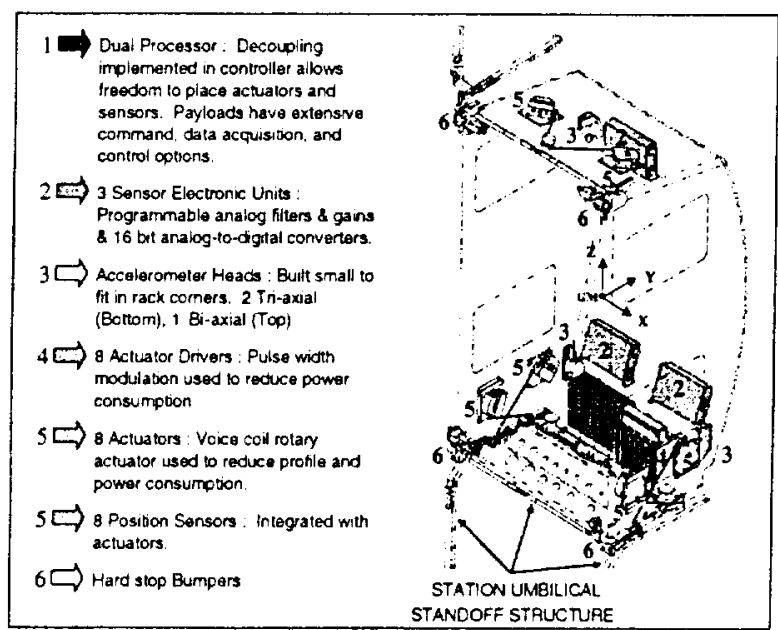

Figure 1: ARIS Payload Rack

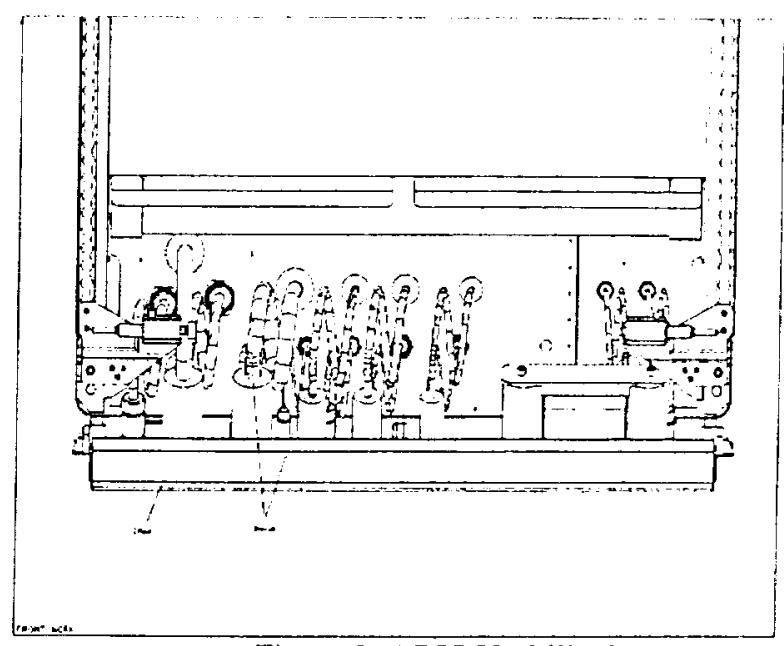

Figure 2: ARIS Umbilicals

The ARIS active control law consists of (a) a low bandwidth position loop that keeps the rack centered in its sway space and prevents the rack from bumping into the station and/or adjacent racks, (b) a higher bandwidth acceleration loop for active vibration attenuation. The stringent microgravity isolation performance desired from ARIS necessitates an integrated approach to the design of the isolation system. Hardware development and control system design cannot be considered to be two standalone activities. A strong interplay between the hardware and control system design is

\footnotetext{
' Corresponding Author
} 
necessary in order to be able to achieve the desired microgravity level isolation performance. Figure 3 shows the "baseline" ARIS isolation performance (dotted line) that corresponds to the existing rack hardware, and compares it to the desired performance (thick solid line).

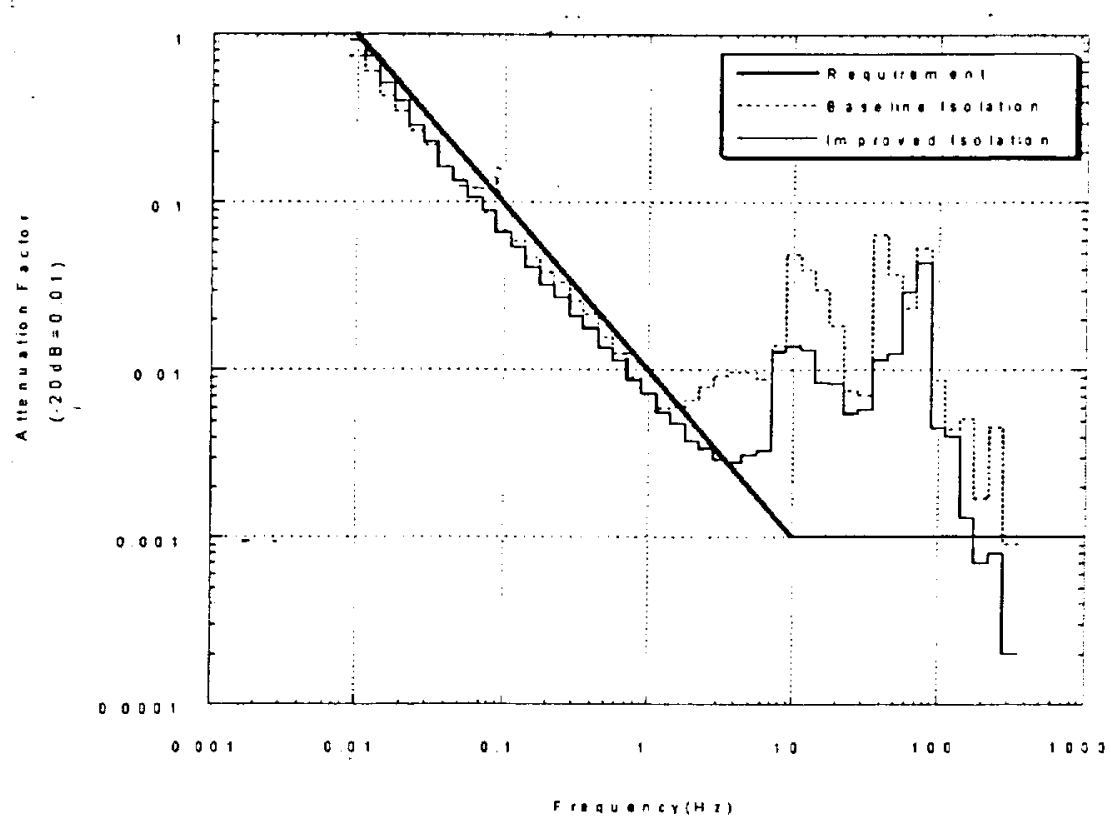

Figure 3: ARIS Closed-loop Isolation Transfer Function

The increase in the transfer function above $2 \mathrm{~Hz}$ is due to the fact that the acceleration loop must roll off in order to gain stabilize the rack flexible modes (which begin above $25 \mathrm{~Hz}$ ). Thus, the rack structural characteristics (flexible modes) place severe limitations on the allowable active acceleration loop bandwidth. This limitation on acceleration loop bandwidth is further compounded by the fact that position sensor bandwidth limits the amount of stiffness compensation that can be accomplished by the position control loop. Higher position loop bandwidth would allow more umbilical stiffness compensation, thereby reducing the required acceleration loop bandwidth necessary to achieve isolation performance. The best situation would be high allowable bandwidths in both the acceleration and position loops. Thus, higher bandwidth position sensors along with approaches to reduce rack structural mode amplification need to be investigated in order to improve isolation performance in the active region. The resonance at $10 \mathrm{~Hz}$ (the passive isolation region) is dominated by the fundamental dynamic resonance of the power umbilicals ${ }^{2}$, while the resonances above $25 \mathrm{~Hz}$ are due to excitation of the rack flexible modes by offboard disturbances transmitted through the umbilical load paths. Thus, an investigation into dynamic characteristics of the umbilicals along with approaches to dampen rack structural vibrations would go a long way in improving isolation performance in the passive region.

\section{Performance In The Active Region}

We begin with hardware improvements and corresponding controller redesign that is aimed at improving isolation performance in the $2-8 \mathrm{~Hz}$ range (active region). As indicated above there are two main options that have potential in this regard:

1. Passive rack damping such as constrained layer damping (CLD) can be used to reduce the structural amplification caused by the rack flexible modes. This would allow a higher acceleration loop bandwidth, thereby improving performance in the active region. Since the acceleration loop rolloff requirement is dictated primarily by the lower frequency $(25-45 \mathrm{~Hz})$ flexible modes, the focus is to target these modes using CLD treatments. CLD treatments capitalize on the inherent damping of highly dissipative visco-elastic materials (VEM) to increase system-level damping performance. A thin layer of VEM is sandwiched between the base structure and a stiff constraining layer. When the structure undergoes cyclic bending, only the surface of the VEM attached to the structure extends and compresses, while

${ }^{2}$ The ARIS system utilizes upto 14 umbilicals, of which only two are power umbilicals. 
the other surface is held in check by the constraining layer. This results in shear strain in the VEM, significantly improving damping efficiency.

The design of a CLD treatment is an iterative, analytical process aimed at determining an optimum configuration (one that produces the most damping for the least weight) within the existing design constraints. The design tool used for this process is the Modal Strain Energy method which relates the modal damping of the system to the modal strain energy distribution and the material loss factors. Finite element (FEM) techniques are used to identify high strain energy areas in the targeted modes (Figure 4) and several damping concepts are investigated. Parametric studies are conducted to determine the optimum VEM (Figure 5) and constraining layer properties, and a final selection is made taking into consideration other factors such as operating temperature, outgassing requirements, and other physical limitations. The final damping design consisted of a 0.04" thick VEM (ISD 112) and a 0.08 " thick Aluminum constraining layer applied to a section of the rack top and side panels. The design goal of $5 \%$ damping was met or surpassed for all targeted modes with minimum weight penalty.

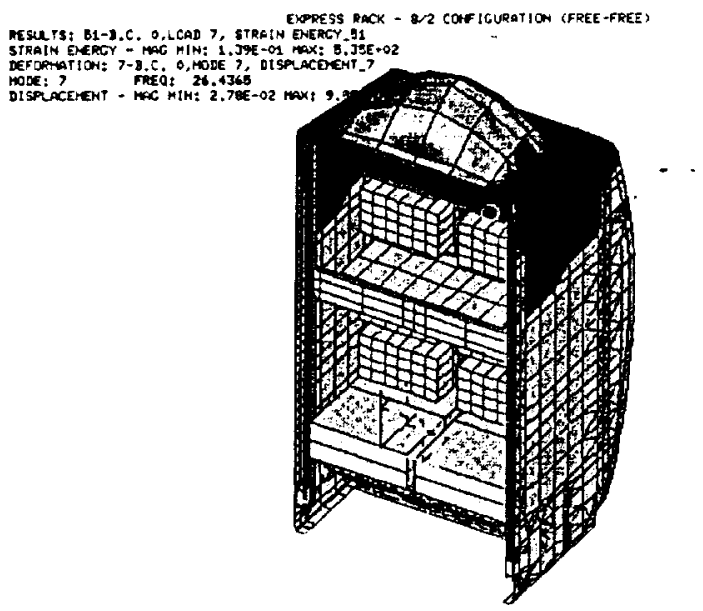

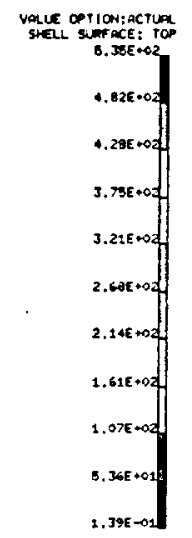

Figure 4: FEM model identification of high strain energy areas

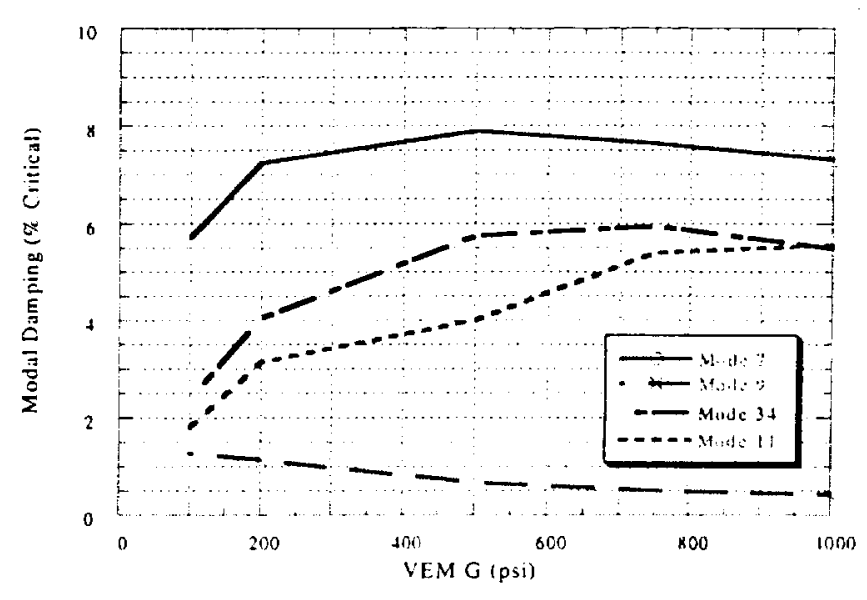

Figure 5: Parametric optimization plot

The efficacy of the CLD treatments is shown in Figures 6 and 7. Figure 6 shows the rack flexible mode amplification without damping treatments, while Figure 7 shows the flexible amplification with the chosen treatment in place. It is clear that the CLD treatment significantly increases the damping on the targeted lower frequency flexible modes.

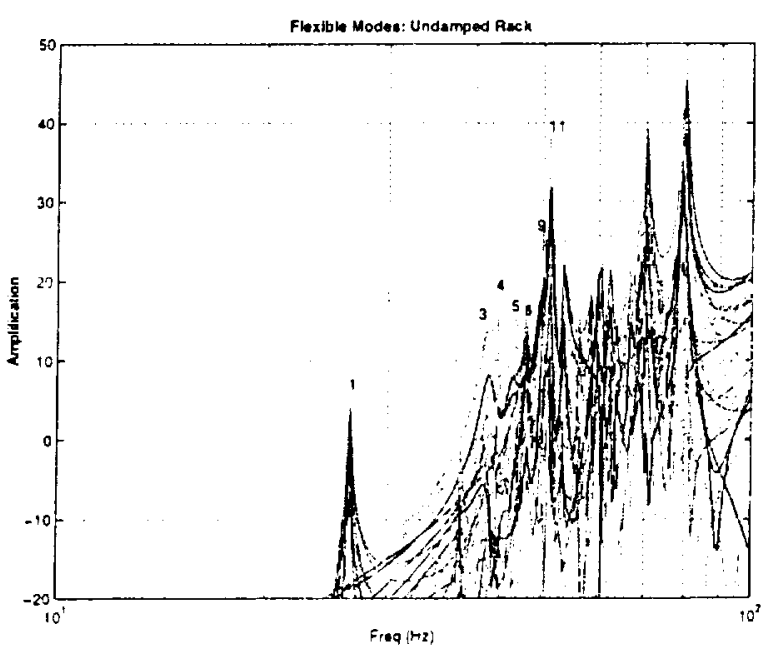

Figure 6: Undamped flexible modes

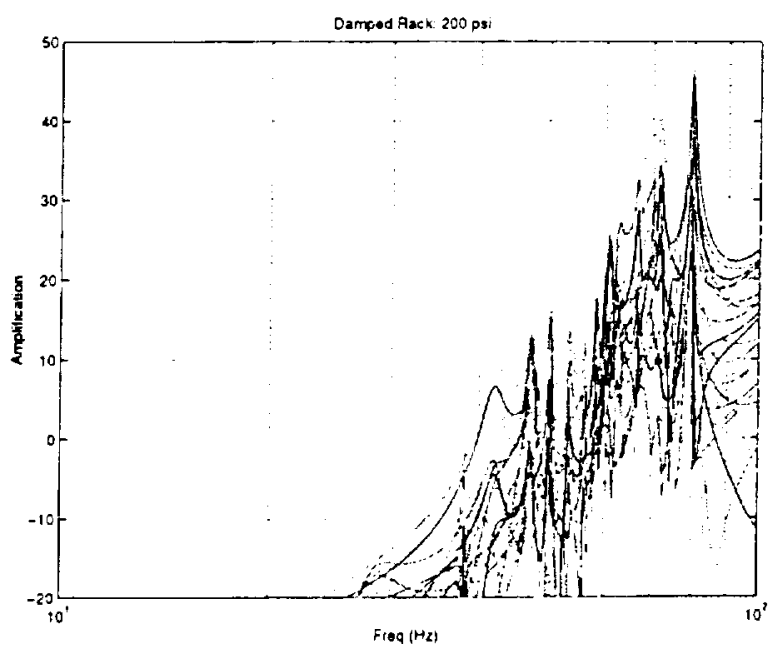

Figure 7: CLD damped flexible modes

2. Increased Position Loop Bandwidth: The present ARIS control law uses the position sensors to close a low frequency $(<0.1 \mathrm{~Hz})$ position tracking loop, as well as to perform umbilical stiffness compensation. The bandwidth of the position loop has been kept low due to the fact that the bandwidth of the position sensors was only conservatively 
known to this point. Recent testing however has shown that the position sensor bandwidth is well beyond $10 \mathrm{~Hz}, \mathrm{a}$ feature that can be exploited to extend the position loop bandwidth, thereby improving isolation in the $2-8 \mathrm{~Hz}$ region.

\section{Performance In The Passive Region}

As discussed above, redesign of the umbilicals in order to reduce their dynamic resonance along with approaches to reduce structural amplification caused by the higher frequency $(>45 \mathrm{~Hz})$ rack modes would help improve isolation performance in the passive (>10 $\mathbf{H z}$ ) frequency range. Higher frequency rack modes tend to be local and as a consequence damping treatments that target these modes would have to be rack specific. Due to the fact that a wide variety of racks with differing structural characteristics are used along with ARIS, damping treatments that target the high frequency modes would have to designed and tested for each rack that is scheduled to be equipped with ARIS. The passive isolation performance improvement that results cannot justify the cost of this endeavor. Hence our investigation into passive performance improvement is limited to improvements in the umbilical dynamic characteristics.

1. Umbilical Dynamic Characteristics: As discussed above, the dynamic resonance of the power umbilicals (at 10 $\mathrm{Hz}$ ) manifests itself in the isolation transfer function shown in Figure 3. Investigations have shown that this resonance is caused by the resonance of the power umbilical loop (see Figure 2). As a consequence, recent testing has been conducted with unlooped power umbilicals. The results are extremely encouraging in that they show that unlooped power umbilicals show virtually no dynamic resonance. However, removing the loop results in an increase in umbilical stiffness (which was the reason the umbilicals were looped in the first place). However, increased stiffness can be compensated for, whereas umbilical resonances which are outside the active bandwidth cannot. Therefore a stiffer unlooped umbilical is preferred over its softer looped counterpart. In addition, research into wire strand/gauge/material and insulation alternatives are underway, in order to understand the effect of these parameters on cable stiffness and dynamics.

\section{$\underline{\text { Main Results }}$}

The improvement in isolation performance that results from the hardware/control design initiatives discussed above are shown in Figure 3 (solid line). The improvement in isolation performance in the $2-8 \mathrm{~Hz}$ region is due to the increased acceleration loop bandwidth that the low frequency CLD treatment allows, as well as due to the increased stiffness compensation that the higher position sensor bandwidth allows. In the passive region the resonance peak at $10 \mathrm{~Hz}$ is significantly lower due to the unlooped power umbilicals. The CLD treatment also results in isolation improvement upto around $45 \mathrm{~Hz}$ (as this was the frequency range that was targeted).

\section{Conclusions}

The paper has discussed the interplay between hardware design and controller design in improving isolation performance of the Active Rack Isolation System. Several initiatives such as higher bandwidth position sensors, reduction in flexible mode amplification via CLD treatments, and improvements in power umbilical dynamic characteristics have been considered, and their impact on achievable isolation performance have been discussed. 